\title{
Modeling and Design of Data Cube for Electricity Bill Deposit System
}

\author{
Vipin Saxena \\ Prof. and Head, Deptt. of Computer Science \\ B. B. Ambedkar University \\ Lucknow, Uttar Pradesh, 226025, INDIA
}

\author{
Sudhir Kumar Singh \\ Deptt. of Computer Science and Engineering \\ Bhagwant University, Ajmer \\ Rajasthan, 305004, INDIA
}

\begin{abstract}
Due to evolution of object-oriented technology, many of the software industries are converting old structured base database into the object oriented database. It is observed from the literature that limited research work is available for the object-oriented database and a well-known modeling language i.e. Unified Modeling Language is not much applied in the field of the object-oriented database, therefore, present work deals with the modeling of a database of a real case study of the electricity bill deposit system of electricity supply to the different houses of various localities of a city. As the size of database grows then it is very difficult to find the desired record from the database and also to keep the record for future use, therefore, a database cube designing technique is used for storing the three important fields of a database and one can get the desired information from the data cube. Sample queries are also performed for validating the designed UML class diagram for electricity billing system.
\end{abstract}

\section{Keywords}

Object-Oriented Database, UML, Class Diagram, Sequence Diagram, Data Cube, Queries.

\section{INTRODUCTION}

Modeling is necessary for getting the solution of the complex problem in easy way. The present work is based upon the latest a object-oriented modeling language which a platform independent language called as Unified Modeling Language (UML). It contains various kinds of diagrams used for the pictorial representation of the complex research problems. This language is not much applied for the object-oriented database, therefore, present work is an attempt in this direction. Let us first describe important research work related to this field.

In the present work a data cube approach is used, therefore, let us first define the data cube. It is a technique that records database in the form of three dimensional cubes. As data is stored inside a cube; it is easily retrievable from the database according to the user's need. Booch[1] has described object oriented analysis and design with various kinds of applications along with various types of diagrams used for the stable software designs.Rumbaugh et al[2] described about Unified Modeling Language for the original developers of the UML. Martin [3] presented UML class diagrams which allow us to denote the static contents of and the relationships between classes. Li [4] presented UML sequence diagrams which are widely used as a behavioral modeling language for interactive systems for their concise and intuitive expression, especially for a few high security systems. Samuel [5] described Sequence diagrams which one of the most widely used UML models in the software industry. Although sequence diagrams are used for modeling the dynamic aspects of the system, they can also be used for model based testing. Existing work does not encompass certain important features of UML 2.0 sequence diagrams. Nakamura et al. [6] presented UML class diagrams representing the static structure of the relations between different concepts existing in a problem are widely used in model-based software development. which no effective measures of a class diagram's understandability yet exist. We have devised quantitative measures of a class diagram's understandability and evaluated their validity. Ali [7] has described we obtained strong correlations between the domain experts' subjective evaluations of the understandability of a class diagram and the measurements of our methods. These results indicate that our measures can effectively quantify the understandability of class diagrams. Chaurasia et al. [8] presented a mobile based electricity bill deposit system through UML with a real case study of electricity department. Harinarayan et al. [9] have presented the applications of greedy algorithms to materialize the cells of data cubes. Cabibbo et al. [10] described a logical approach for the multidimensional database. Lehner et al. [11] have proposed the various normal forms for multidimensional database with key concepts in On Line Analytical Processing (OLAP) which measures the facts and data cubes. Vanea et al. [12] described aggregated data which has the potential to offer great knowledge and can helpful for decision makers in their most important activities. Xie et al. [13] presented a novel technique to reduce the data cube size by dividing the cube into chunks and using this reduced data cube to provide answers to the direct queries. Han et al [14] described mining techniques for the complex data and algorithms. Chen [15] has described a new database structure model which organizes the multimedia data in a multi-dimensional data cube. Clustered data are stored in instance table in the data cube. A corresponding leading data's are stored in dimension table. When querying, a leading data is gained from dimension table firstly, followed by a k-nearest neighbor query from the corresponding category, and then the retrieval results are returned.

The present paper deals with the modeling of a real case study and design of data cube for the power corporation department of India where a large amount of bills are generated daily and it is very difficult to keep record for future use, therefore a technique of three dimensional data is presented for storing the three important fields inside the data cubes. Sample queries are also performed for validating the UML designs and the desired information from the data cubes. For modeling purpose, UML class and sequence diagrams are designed. 


\section{UML MODELING FOR BILLING SYSTEM}

\subsection{A UML Class Diagram}

The creation of table T1 in object oriented database is the first step. T1 includes four fields namely Serial Number (SNo), Name, DueDate and Amount as represented in figure 1. A group of 100 data is stored in the Table T1. Data is stored in data cube using On Line Analytical Processing (OLAP) cube software. OlAP cube software commands the object-oriented data base in Table T1. A cube must contain at least two dimensions and one measure. This results in the creation of a data cube. Though date is stored in the form of cube, the output can only be seen in the form of graphs and tables. Data cube supports the storage of millions data. Each data stored in a sequence form. OLAP database has other name which is called as Multi-dimensional database (MDDB). OLAP technology has divided in two forms. First name is Relational OLAP (ROLAP) and second name is Multidimensional OLAP (MLOAP). The main work of MLOAP is to store data directly in n-dimensional array while ROLAP stored in the form of relational database.

\begin{tabular}{|l|}
\hline \multicolumn{1}{|c|}{ T1 } \\
\hline -SNo \\
-Name \\
-DueDate \\
-Amount \\
\hline +Customer_record() \\
\hline
\end{tabular}

\section{Fig 1: UML Class Definition of T1}

The above class diagram shows the static behavior of system. Let us design the class diagram for billing system as shown in figure 2. The class diagram has four persistent classes Bill, Mobile, Elec_Office, Online Server and one transient class Customer. These classes are connected to each other using relationship. In this paper, Online Server sends the electric bill data on the user's mobile device. Customer is a class which has attributes like customer_id, customer_name, customer_street, customer_age, customer_city. Bill is a class of bill_no, bill_amount, person_id, deposit_date. Mobile is a class of mobile_comp, mobile_model, mobile_no, iemi_no. Elec_Office is a class of office_address, office_city, office_code and all personal record stores in electrical office. Online server sends the bill on user mobile device.

\subsection{A UML Sequence Diagram}

Sequence diagram is a part of UML. The work of sequence diagram is to send message to the target path. Sequence diagram finds the sender and receiver. Sender sends the message on target path then receiver shows the acknowledgement to sender. Sequence diagram shows two axis horizontal and vertical axes. Vertical line shows the lifeline and horizontal line shows the message communication.

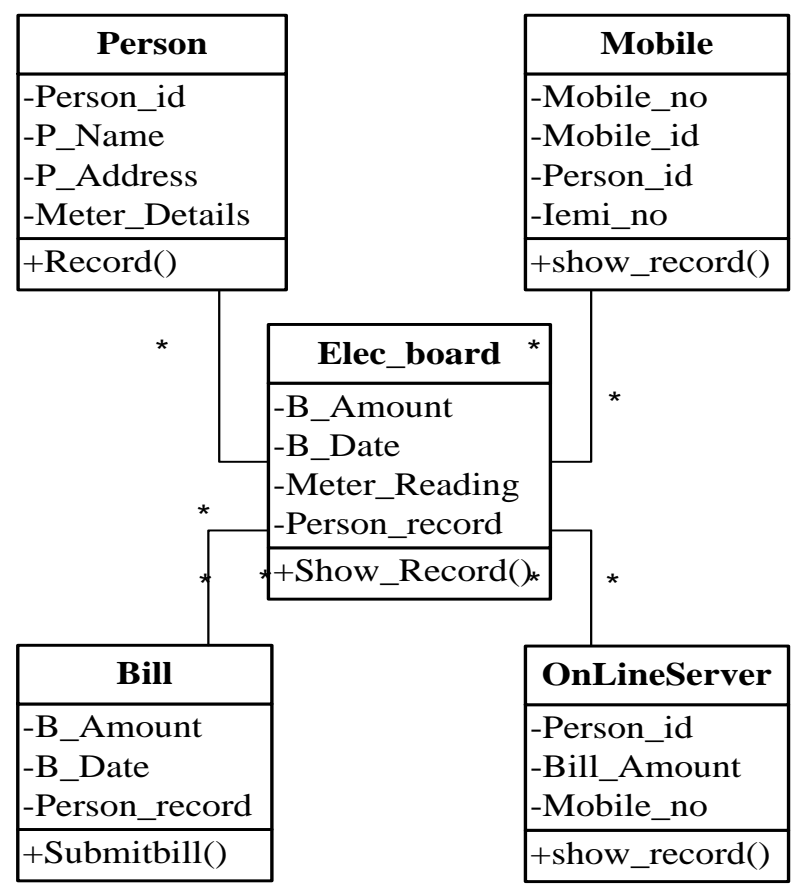

Fig 2: Class Diagram of Electrical Bill Deposit System

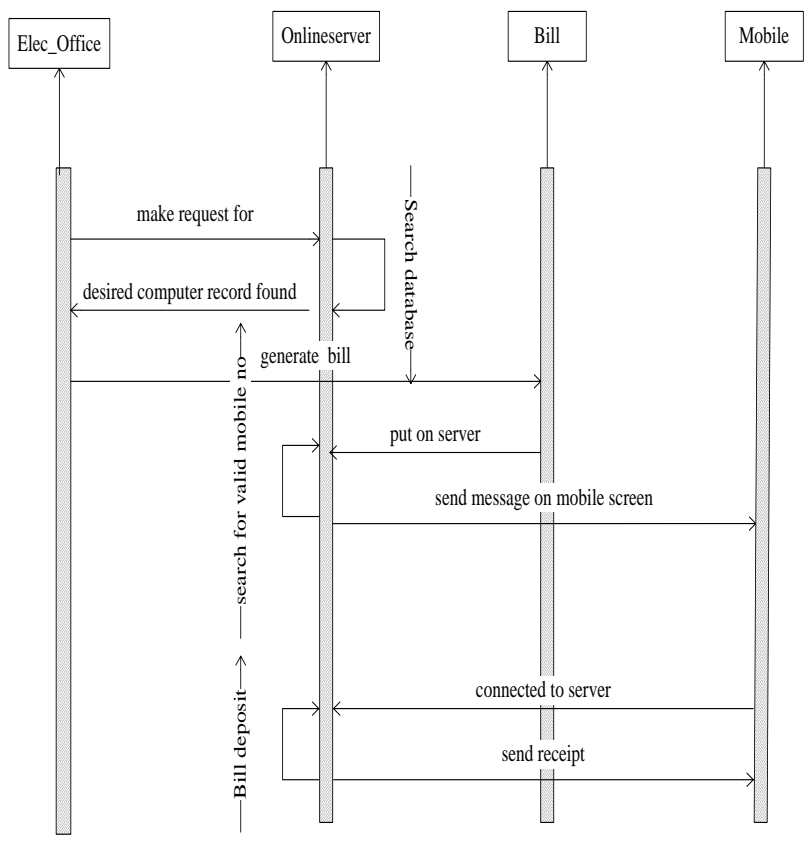

Fig 3: Sequence Diagram Electrical Bill Deposit System

Sequence diagram shows four objects Elec_office, Online server, Bill and Mobile. The diagram shows that computer sends the request to server and server passes the message on particular mobile number. Sequence diagram represents graphical symbol. In this diagram, box and attached arrow called objective lifeline. Forward arrow called the message and reverse arrow called the message return. Elec_office stores the million data in the electricity bill record. In this sequence diagram, Elec_office makes a request for desired computer and search the record inside the database. Database founds the record after generation of bill, thereafter, Bill sends on the Mobile device of customer after searching the valid 
mobile number. found the valid mobile no connected to server. After connect to server bill show in mobile device

\section{DESIGN OF OLAP CUBE}

Online Analytical Processing (OLAP) cubes are specially designed for storing the database. OLAP cube stores million data in the form of multidimensional. OLAP cube are of two categories i.e. dimension and measure. The main advantage of designing of cube is that data is easily retrieved in comparison of other database. Data cube supports the division of data into three axes i.e. $\mathrm{X}, \mathrm{Y}$ and $\mathrm{Z}$. It also supports the decision support system. Though data is stored in the form of cube, the output can only be seen in the form of graphs and tables. Each data is stored in a sequence form. The other name of OLAP database is Multi-Dimensional database (MDDB). OLAP technology has divided in two forms. First name is Relational OLAP (ROLAP) and second name is Multidimensional OLAP (MLOAP). The main work of MLOAP is to store data directly inside $n$-dimensional array. The main work of ROLAP is to store the Relational database. OLAP cube is generally used for business purpose and for calculation of the company profit and loss for financial year. The general representation of OLAP cube is shown below in figure 4 .

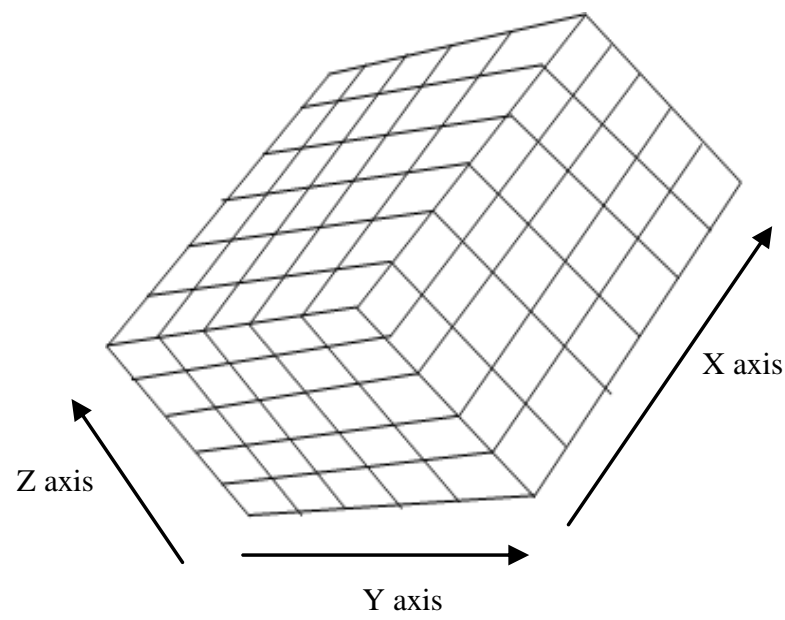

Fig 4: General Representation of OLAP Data Cube

On the basis of above, OLAP cube is created by taking three axes as Mobile_no, Person-id and Bill according to X, Y and $\mathrm{Z}$ axes as shown in figure 5. A sample data of 100 records of electricity bill is generated. OLAP cube software calls the table T1 and creates the data cube D1. The sample data is stored inside the data cube D1. Queries are performed on data cube D1 and results are shown below. Object-oriented database is much better than the relational database. The reason is that the object-oriented database stabilizes the relation between objects but not in the table. Data cube D1 supports decision support system. The data in data cube D1 can be easily retrieved. The different queries are given below:

\section{Sample Query-I}

This database query is performed in oracle $10 \mathrm{G}$ version. This table stores the person record. Person record table found the Person_id(P_id), Person_name(P_name) and Person_address (P_address).

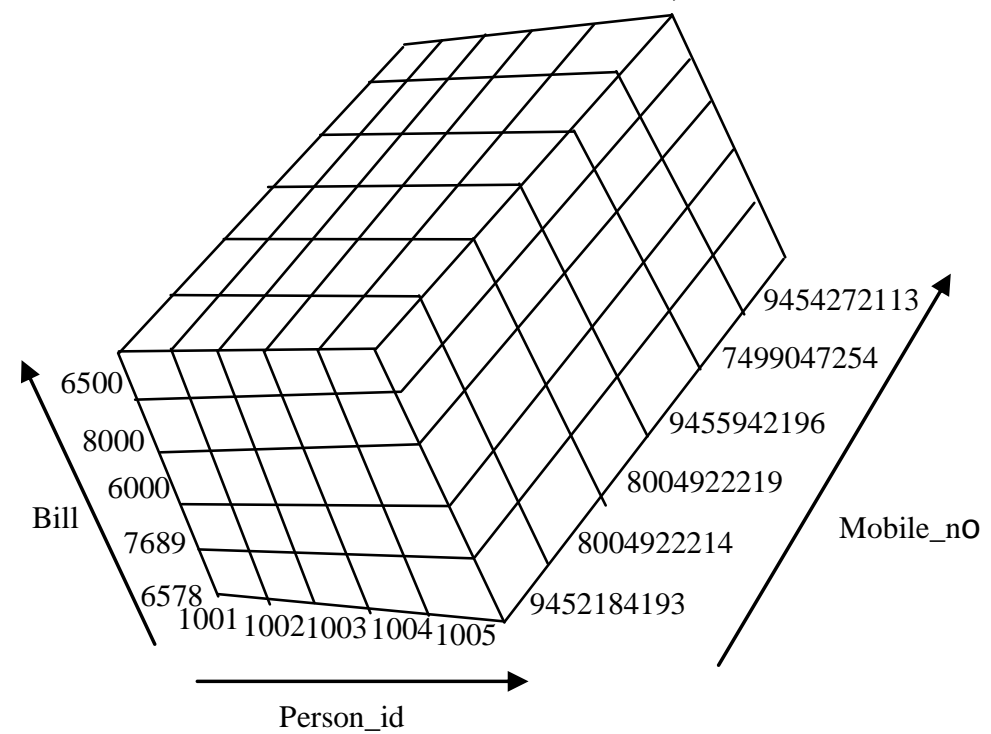

Fig 5: Data Cube D1 for Electricity Billing System

SQL>create table person (p_id number(10),p_name varchar2(15),p_address varchar2(15));

$\mathrm{SQL}>$ select * from person;

This table stores the person record as shown below:

\begin{tabular}{|c|c|c|}
\hline P_id & P_name & P_address \\
\hline 1001 & Singh & Lucknow \\
\hline 1002 & Rama & Lucknow \\
\hline 1003 & Shivendre & Lucknow \\
\hline 1004 & Arun & Lucknow \\
\hline 1005 & Praveen & Lucknow \\
\hline 1006 & Sudhir & Lucknow \\
\hline 1007 & Sanjeev & Lucknow \\
\hline 1008 & Pranay & Lucknow \\
\hline
\end{tabular}

\section{Sample Query-II}

In this database query, bill_record table keeps the bill_id (b_id) and person_id (p_id) record as defined below:

SQL>create table bill_rec (b_amount number(10),b_id number(10),p_id number(10));

SQL> select * from bill_rec;

This table stores the bill_rec data as shown below:

\begin{tabular}{|c|c|c|}
\hline B_Amount & B_id & P_id \\
\hline 6578 & 111 & 1001 \\
\hline 6000 & 112 & 1002 \\
\hline 7689 & 113 & 1003 \\
\hline 8000 & 114 & 1004 \\
\hline 6500 & 115 & 1005 \\
\hline 7800 & 116 & 1006 \\
\hline 8000 & 117 & 1007 \\
\hline 8210 & 118 & 1008 \\
\hline
\end{tabular}




\section{Sample Query-III}

Mobile database table stores the customer mobile_no and person_id( $\left.\mathrm{p} \_i d\right)$. The query is given below:

SQL>create table mobile (mobile_no number (15), p_id number (10), iemi_no number (10));

Table created.

SQL> select * from mobile_rec;

This table stores the mobile_rec as shown below:

\begin{tabular}{|c|c|}
\hline Mobile_no & P_id \\
\hline 9452184193 & 1001 \\
\hline 8004922214 & 1002 \\
\hline 8004922219 & 1003 \\
\hline 9455942196 & 1004 \\
\hline 9695328477 & 1005 \\
\hline 7499047254 & 1006 \\
\hline 9454272113 & 1007 \\
\hline 930556624 & 1008 \\
\hline
\end{tabular}

\section{Sample Query-IV}

This database table sends the electrical bill on the user's mobile no.

\section{SQL>select}

person.p_id,mobile_rec.mobile_no,bill_rec.b_amount from person,mobile_rec,bill_rec where person. $\mathrm{P}$ _id=mobile_rec. P_id and person. $P \_i d=b i l l \_r e c . P \_i d$;

This table sends the amount of bill on user's mobile device as shown below:

\begin{tabular}{|c|c|r|}
\hline P_id & Mobile_no & B_amount \\
\hline 1001 & 9452184193 & 6578 \\
\hline 1002 & 8004922214 & 6000 \\
\hline 1003 & 8004922219 & 7689 \\
\hline 1004 & 9455942196 & 8000 \\
\hline 1005 & 7499047254 & 6500 \\
\hline 1006 & 9454272113 & 7800 \\
\hline 1007 & 9454272113 & 8000 \\
\hline 1008 & 9305566124 & 8210 \\
\hline
\end{tabular}

\section{CONCLUDING REMARKS}

In the present work, object-oriented database is created which is better than the relational database which was used in the structured approach earlier. A UML class model is designed for the execution of several sample queries and observed that model works well for designed database which was also stored inside the data cubes for the large database of electrical billing system. The desired information is shown on the mobile device so that users could deposit the bill of electricity as per specified last date of bill deposit. When the database is large, then the present work can be extended for the designing of association rules for the removal of unwanted database.

\section{REFERENCES}

[1] Booch, G., "Object Oriented Analysis and Design with Application", $2^{\text {nd }}$ Edition, Addison Wesley 1994.

[2] Rambaugh, J., Jacobson, I and Booch, G., "The Unified Modeling Language User Guide", Addison Wesley, 1999.

[3] Martin, F., "UML Distilled: A Brief Guide to the Standard Object Modeling Language", $3^{\text {rd }}$ Edition, Addison-Wesley, Boston, 2000.

[4] Li, M., "Approach to Formalizing UML Sequence Diagrams", Presented IEEE, Page 1-4, 28 to 29 May, 2011.

[5] Samuel, P., "Test Sequence Generation from UML Sequence Diagrams", presented IEEE, Page 879-887, 6 Aug. 2008.

[6] Nakamura, Sakamoto, Y., Inoue, K., Washizaki, H. and Fukazawa, Y., "Evaluation of Understandability of UML Class Diagrams by Using Word Similarity", Presented in IEEE, Page 178-187, 20 Dec 2011.

[7] Ali, N., "A Design of an Assessment System for UML Class Diagram", This Paper appears in Computational Science and its Applications, 2007 ICCSA 2007.International Conference on IEEE Page 539, Aug. 2007.

[8] Chaurasia and Saxena, V., "Mobile Based Electricity Bill Deposit System Through UML, " Journal of Software Engineering and Applications, Vol.4(3), 187-190, 2011.

[9] Harinarayan, V., Rajaraman, A., and Ulman, J., "Efficient Implementation of Data Cube via Materialized Views", In Proceedings of ACM SIGMOD Conference Vol.(25), Page No 205-216, Montreal, Canada 1996.

[10] Cabibbo, L., and Torlone, R., "A Logical Approach to Multidimensional Database", $6^{\text {th }}$ International Conference on Extending Database technology (EDBT, 98, Valencia, Spain), G. Alonso (eds.), Lncs, Vol.1377, Springer, 1998

[11] Lehner, W., Albrecht, J., and Wedekinds, H., " Normal Forms for Multidimensional Database", In Proceedings of the $10^{\text {th }}$ International Conference on Scientific and Statistical Data Management (SSDBM'98),Italy,1998.

[12] Vanea, A., and Potolea, R., "A Method for Dynamically Creating Data Cube Structure", IEEE International Conference Appeared Intelligent Computer Communication and Processing (ICCP), Date 25-27 Aug 2011.

[13] Xie, Z., and Guang, J., " The Algorithm of reducing Data Cube Rapidly", Published in: Management and Service Science, 2009. MASS '09. International Conference, Date 20-22 Sept 2009.

[14] Han, J., and Kamber, M., "Data Mining: Concepts and Technique", $2^{\text {nd }}$ Edition, Morgan Kaufmann Publishers, March 2006.

[15] Chen, M., "Multimedia Database Retrieval Based on Data Cube", Proceedings of the IEEE, Page 1265-1269, 2008. 